\title{
Liouville Decoherence in a Model of Flavour Oscillations in the presence of Dark Energy
}

\author{
Nick Mavromatos and Sarben Sarkar \\ Department of Physics \\ King's College London \\ Strand, London WC2R 2LS, UK
}

\begin{abstract}
We study in some detail the master equation, and its solution in a simplified case modelling flavour oscillations of a two-level system, stemming from the Liouvillestring approach to quantum space time foam. In this framework we discuss the appearance of diffusion terms and decoherence due to the interaction of low-energy string matter with space-time defects, such as D-particles in the specific model of "D-particle foam", as well as dark energy contributions. We pay particular attention to contrasting the decoherent role of a cosmological constant in inducing exponential quantum damping in the evolution of low-energy observables, such as the probability of flavour oscillations, with the situation where the dark energy relaxes to zero for asymptotically large times, in which case such a damping is absent. Our findings may be of interest to (astrophysical) tests of quantum space-time foam models in the not-so-distant future.
\end{abstract}




\section{Introduction and Motivation}

In recent years there has been a debate on whether microscopic black holes can induce quantum decoherence at a microscopic level. The presence of quantum-fluctuating microscopic horizons, of radius of the order of Planck length $\left(10^{-35} \mathrm{~m}\right)$, may give space-time a "foamy" structure, causing decoherence of matter propagating in it. In particular, it has been suggested [1] that such Planck-scale black holes and other topological fluctuations in the space-time background cause a breakdown of the conventional S-matrix description of asymptotic scattering in local quantum field theory. This may lead to experimentally testable predictions, at least in principle, for instance as regards the so-called sensitive particle-physics probes of quantum mechanics [2, 3, 4].

It must be pointed out that this suggestion is invalidated if there is holography in quantum gravity [5], such that any information of quantum numbers of matter, that at first sight appears to be lost into the horizon, is somehow reflected back on the horizon surface, thereby maintaining quantum coherence. This may happen, for instance, in some highly supersymmetric effective theories of strings [6], which however do not represent realistic low-energy theories of quantum gravity. Supersymmetry breaking complicates the issue, spoiling complete holography. Recently, S. Hawking, inspired by the above recent ideas in string theory, has also argued against the loss of coherence in a Euclidean quantum theory of gravity. In such a model, summation over trivial and non-trivial (black-hole) space-time topologies in the path over histories makes an asymptotic observer "unsure" as to the existence of the microscopic black hole fluctuation thus resulting in no loss of quantum coherence. However, this sort of argument is plagued not only by the Euclidean formalism, with its concomitant problems of analytic continuation, but also by a lack of a concrete rigorous proof, at least up to now.

Therefore, for our purposes we still consider the matter of quantum-gravity-induced decoherence as wide open and worthy of further phenomenological exploitation. This is the point of view we shall take in this work. We shall restrict ourselves to a specific framework for analyzing decoherent propagation of low-energy matter in foamy spacetime backgrounds in the context of string theory [7, 8, the so-called Liouville-string [9] decoherence [10]. Our motivation for using string theory is that it appears at present to be the best controlled theory of quantum gravity available to date. In this respect we also mention that there are other interesting approaches to quantum space-time foam, which also lead to experimental predictions, for instance the "thermal bath" approach advocated in [11], according to which the foamy gravitational environment may behave as a thermal bath, inducing decoherence and diffusion in the propagating matter, as well as quantum damping in the evolution of low-energy observables, features which are, at least in principle, testable experimentally. As we shall see later on, similar behaviour is exhibited by the specific models of foam that we study here, which may characterize modern versions of string theory [8], specifically the D-particle foam model of [12, 13], based on point-like membrane defects in space time (D-particles).

In the presence of decoherence the S-matrix of the effective low-energy field theory would then have to be replaced by a linear non-factorisable superscattering operator $/ S$ 
relating initial and final-state density matrices [1]

$$
\rho_{\text {out }}=\not S \rho_{\text {in }}
$$

If this is correct then the usual formulation of quantum mechanics has to be modified. Arguments have been put forward for this modification of the Liouville equation to take the form [2]

$$
\partial_{t} \rho=\frac{i}{\hbar}[\rho, H]+\not \supset H \rho .
$$

Equations of this form are encountered in the description of the time evolution of the state of open quantum mechanical systems where $\not h \rho$ has a Lindblad form [14. In such systems observable degrees of freedom are coupled to unobservable components which are effectively integrated over. Initial pure states evolve into mixed ones and so

$$
\not S \neq S S^{\dagger}
$$

where $S=e^{i H t}$. In these circumstances Wald [15] has shown that CPT is violated, at least in its strong form, i.e. there is no unitary invertible operator $\Theta$ such that

$$
\Theta \bar{\rho}_{\text {in }}=\rho_{\text {out }} .
$$

Such considerations have more recently again come to the fore because of current neutrino data including LSND data [16. There is experimental evidence, that the neutrino has mass which leads to neutrino oscillations. However LSND results appear consistent with the dominance of anti-neutrino oscillations $\bar{\nu}_{e} \rightleftarrows \bar{\nu}_{\mu}$ over neutrino oscillations. In particular, provided LSND results turn out to be correct, which is unclear at present, there is evidence for CPT violation. It has been suggested recently [17] that Planck scale quantum decoherence may be a relevant contribution to the CPT violation seen in the experiments of LSND. Other examples of flavour oscillating systems with quite different mass scales are furnished by $B \bar{B}$ and $K \bar{K}$ systems [3]. The former, because of the large masses involved, provides a particularly sensitive system for investigating the Planck scale fluctuations embodied by space-time foam. In all these cases experiments, such as CPLEAR [4], provide very low bounds on CPT violation which are not inconsistent with dimensional analysis estimates for the magnitudes of effects from space-time foam. These systems have been analyzed within a dynamical semigroup approach to quantum Markov processes. Once the framework has been accepted then a master equation for finite-dimensional systems ensued which can be characterized by a small set of parameters. This approach is somewhat phenomenological and is primarily used to fit data. Consequently it is important to obtain a better understanding of the nature of decoherence from a more fundamental viewpoint.

An additional, and perhaps more plausible [18, reason for considering quantum decoherence models of quantum gravity, comes from recent astrophysical evidence on a currentera acceleration of our Universe. Indeed, observations of distant supernovae [19], as well as WMAP data [20] on the thermal fluctuations of the cosmic microwave background $(\mathrm{CMB})$, indicate that our Universe is at present in an accelerating phase, and that $73 \%$ of 
its energy-density budget consists of an unknown substance, termed Dark Energy. Best-fit models of such data include Einstein-Friedman-Robertson-Walker Universes with a nonzero cosmological constant. However, the data are also currently compatible with (cosmic) time-dependent vacuum-energy-density components, relaxing asymptotically to zero 21. In colliding brane-world models the dark energy component of the Universe is considered to be a non-equilibrium energy density of the (observable) brane world [22, 12]. This density is identified with the central charge surplus of the supercritical $\sigma$-models describing the (recoil) string excitations of the brane after the collision. The relaxation of the dark-energy density component can be a purely stringy feature of the logarithmic conformal field theory [23] describing the D-brane recoil [24] in a (perturbative) $\sigma$-model framework. We shall discuss below how the theory of non-critical strings deals with the twin problems of cosmological constant, and dark energy. It is worth pointing out that the non-critical (Liouville) string [9] provides a rather unified formalism for dealing not only with Universes with a non-zero cosmological constant in string theory, but in general with decoherent quantum space-time foam backgrounds, that include microscopic quantum-fluctuating black holes [10].

\section{Liouville decoherence: general formalism}

Given the very limited understanding of gravity at the quantum level, the analysis of modifications of the quantum Liouville equation implied by non-critical strings can only be approximate and should be regarded as circumstantial evidence in favour of the dissipative master equation. In the context of two-dimensional toy black holes 25] and in the presence of singular space-time fluctuations there are believed to be inherently unobservable delocalised modes which fail to decouple from light (i.e. the observed) states. The effective theory of the light states which are measured by local scattering experiments can be described by a non-critical Liouville string. This results in an irreversible temporal evolution in target space with decoherence and associated entropy production.

The following master equation for the evolution of stringy low-energy matter in a nonconformal $\sigma$-model can be derived [10]

$$
\partial_{t} \rho=i[\rho, H]+: \beta^{i} \mathcal{G}_{i j}\left[g^{j}, \rho\right]:
$$

where $t$ denotes time (Liouville zero mode), the $H$ is the effective low-energy matter Hamiltonian, $g^{i}$ are the quantum background target space fields, $\beta^{i}$ are the corresponding renormalization group $\beta$ functions for scaling under Liouville dressings and $\mathcal{G}_{i j}$ is the Zamolodchikov metric [26, 27] in the moduli space of the string. The double colon symbol in (5) represents the operator ordering : $A B:=[A, B]$. The index $i$ labels the different background fields as well as space-time. Hence the summation over $i, j$ in (5) corresponds to a discrete summation as well as a covariant integration $\int d^{D+1} y \sqrt{-g}$ where $y$ denotes a set of $(D+1)$-dimensional target space-time co-ordinates and $D$ is the space-time dimensionality of the original non-critical string. 


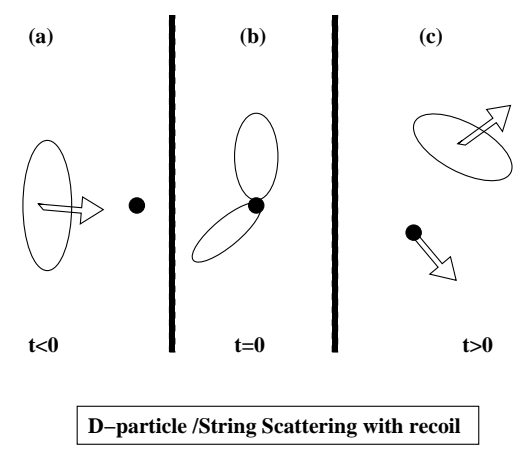

Figure 1: Schematic picture of the scattering of a string matter state on a D-particle, including recoil of the latter. The sudden impulse at $t=0$, implies a back reaction onto the space time, which is described by a logarithmic conformal field theory. The method allows for the perturbative calculation of the induced space-time distortion due to the entangled state in (b).

\subsection{D-particle Foam and Master Equation}

The discovery of new solitonic structures in superstring theory [8] has dramatically changed the understanding of target space structure. These new non-perturbative objects are known as D-branes and their inclusion leads to a scattering picture of space-time fluctuations. The study of D-brane dynamics has been made possible by Polchinski's realization 8 that such solitonic string backgrounds can be described in a conformally invariant way in terms of world sheets with boundaries. On these boundaries Dirichlet boundary conditions for the collective target-space coordinates of the soliton are imposed. Heuristically, when low energy matter given by a closed (or open) string propagating in a $(D+1)$-dimensional space-time collides with a very massive D-particle embedded in this space-time, the Dparticle recoils as a result. Since there are no rigid bodies in general relativity the recoil fluctuations of the brane and their effectively stochastic back-reaction on space-time cannot be neglected.

Based on these considerations, a model for a supersymmetric space-time foam has been suggested in [12]. The model is based on parallel brane worlds (with three spatial large dimensions), moving in a bulk space time which contains a "gas" of D-particles. The number of parallel branes used is dictated by the requirements of target-space supersymmetry in the limit of zero-velocity branes. One of these branes represents allegedly our Observable Universe. As the brane moves in the bulk space, D-particles cross the brane in a random way. From the point of view of an observer in the brane the crossing D-particles will appear as space-time defects which flashing on and off, i.e. microscopic space-time fluctuations. This will give the four-dimensional brane world a "D-foamy" structure.

Closed and open strings propagate on the brane. Each time these strings cross with a Dparticle, there is a possibility of being attached to it, as indicated in Fig. 1. The entangled state causes a back reaction onto the space-time, which can be calculated perturbatively using the formalism of logarithmic conformal field theory [24]. Details are reviewed in 
Appendix B.

Using this model for space-time fluctuations we will obtain an expression for the induced space-time distortion as a result of D-particle recoil. The set $\left\{g^{i}\right\}$ includes the graviton fields $g_{M N}$ where $M$ and $N$ are target space-time indices and in the weakly coupled string limit we can show that:

$$
g_{\ell m}=\delta_{\ell m}, g_{00}=-1, g_{0 \ell}=\varepsilon\left(\varepsilon y_{\ell}+u_{\ell} t\right) \Theta_{\varepsilon}(t), \ell, m=1, \ldots, D
$$

where the suffix 0 denotes temporal (Liouville) components and

$$
\begin{aligned}
\Theta_{\varepsilon}(t) & =\frac{1}{2 \pi i} \int_{-\infty}^{\infty} \frac{d q}{q-i \varepsilon} e^{i q t}, \\
u_{\ell} & =\left(k_{1}-k_{2}\right)_{\ell},
\end{aligned}
$$

with $k_{1}\left(k_{2}\right)$ the momentum of the propagating closed-string state before (after) the recoil; $y_{n}$ are the spatial collective coordinates of the D particle and $\varepsilon^{-2}$ is identified with the target Minkowski time $t$ for $t \gg 0$ after the collision [24] (see Appendix B). These relations have been calculated for non-relativistic branes where $u_{n}$ is small and require the machinery of logarithmic conformal field theory. Now for large $t$, to leading order,

$$
g_{0 \ell} \simeq \bar{u}_{\ell} \equiv \frac{u_{\ell}}{\varepsilon} \propto \frac{\Delta p_{\ell}}{M_{P}}
$$

where $\Delta p_{\ell}, \ell=1, \ldots, D$, is the momentum transfer during a collision and $M_{P}$ is the Planck mass (actually, to be more precise, $M_{P}=M_{s} / g_{s}$, where $g_{s}<1$ is the (weak) string coupling, and $M_{s}$ is a string mass scale); so $g_{0 i}$ is constant in space-time but depends on the energy content of the low energy particle and $R_{M N}=0$. Since we are interested in fluctuations of the metric the indices $i$ will correspond to the pair $M, N$.

However, as already mentioned in the introduction, recent astrophysical observations from different experiments all seem to indicate that $73 \%$ of the energy of the Universe is in the form of dark energy. Best fit models give the positive cosmological constant EinsteinFriedman Universe as a good candidate to explain these observations. For such de Sitter backgrounds $R_{M N} \propto \Omega g_{M N}$ with $\Omega>0$ a cosmological constant. Also in a perturbative derivative expansion (in powers of $\alpha^{\prime}$ where $\alpha^{\prime}=l_{s}^{2}$ is the Regge slope of the string and $l_{s}$ is the fundamental string length) in leading order

$$
\beta_{\mu \nu}=\alpha^{\prime} R_{\mu \nu}=\alpha^{\prime} \Omega g_{\mu \nu}
$$

and

$$
\mathcal{G}_{i j}=\delta_{i j}
$$

This leads to

$$
\partial_{t} \rho=i[\rho, H]+\alpha^{\prime} \Omega: g_{M N}\left[g^{M N}, \rho\right]:
$$

For a weak-graviton expansion about flat space-time, $g_{M N}=\eta_{M N}+h_{M N}$, and

$$
h_{0 \ell} \simeq \bar{u}_{\ell} \equiv \frac{u_{\ell}}{\varepsilon} \propto \frac{\Delta p_{\ell}}{M_{P}} .
$$


If an antisymmetric ordering prescription is used, then the master equation for low energy string matter assumes the form

$$
\partial_{t} \rho_{\text {Matter }}=i\left[\rho_{\text {Matter }}, H\right]-\Omega\left[h_{0 \ell},\left[h^{0 \ell}, \rho_{\text {Matter }}\right]\right]
$$

( when $\alpha^{\prime}$ is absorbed into $\Omega$ ). In view of the previous discussion this can be rewritten as

$$
\partial_{t} \rho_{\text {Matter }}=i\left[\rho_{\text {Matter }}, H\right]-\Omega\left[\bar{u}_{\ell},\left[\bar{u}^{\ell}, \rho_{\text {Matter }}\right]\right] .
$$

thereby giving the master equation for Liouville decoherence in the model of a D-particle foam with a cosmological constant.

\section{Destruction of interference}

The master equation which has been derived is of relevance to the study of general features of gravity induced decoherence. The above D-particle inspired approach deals with possible non-perturbative quantum effects of gravitational degrees of freedom. The analysis is totally unrelated to the phenomenology of dynamical semigroups which does not embody specific properties of gravity. Indeed the phenomenology is sufficiently generic that other mechanisms of decoherence such as the MSW effect can be incorporated within the same framework. Consequently an analysis which is less generic and is related to the specific decoherence implied by non-critical strings is necessary.

It is sufficient to study a massive non-relativistic particle propagating in one dimension to establish qualitative features of D-particle decoherence. The environment will be taken to consist of both gravitational and non-gravitational degrees of freedom; hence we will consider a generalisation of quantum Brownian motion for a particle which has additional interactions with D-particles. This will allow us to compare qualitatively the decoherence due to different environments. The non-gravitational degrees of freedom in the environment (in a thermal state) are conventionally modelled by a collection of harmonic oscillators with masses $m_{n}$, frequency $\omega_{n}$ and co-ordinate operator $\widehat{q}_{n}$ coupled to the particle coordinate $\widehat{x}$ by an interaction of the form $\sum_{n} g_{n} \widehat{x} \widehat{q}_{n}$. The master equation which is derived can have time dependent coefficients due to the competing timescales, e.g. relaxation rate due to coupling to the thermal bath, the ratio of the time scale of the harmonic oscillator to the thermal time scale etc. However an ab initio calculation of the timedependence is difficult to do in a rigorous manner. It is customary to characterise the nongravitational environment by means of its spectral density $I(\omega)\left(=\sum_{n} \delta\left(\omega-\omega_{n}\right) \frac{g_{n}^{2}}{2 m_{n} \omega_{n}}\right)$. The existence of the different time scales leads in general to non-trivial time dependences in the coefficients in the master equation which are difficult to calculate in a rigorous manner 28]. The dissipative term in (14) involves the momentum transfer operator due to recoil of the particle from collisions with D-particles (8). This transfer process will be modelled by a classical Gaussian random variable $r$ which multiplies the momentum operator $\widehat{p}$ for the particle:

$$
\overline{u_{x}} \quad \rightarrow \quad \frac{r}{M_{P}} \widehat{p}
$$


Moreover the mean and variance of $r$ are given by

$$
\langle r\rangle=0, \quad \text { and } \quad\left\langle r^{2}\right\rangle=\sigma^{2} .
$$

On amalgamating the effects of the thermal and D-particle environments, we have for the reduced master equation for the matter (particle) density matrix $\rho$ (on dropping the Matter index)

$$
i \frac{\partial}{\partial t} \rho=\frac{1}{2 m}\left[\widehat{p}^{2}, \rho\right]-i \Lambda[\widehat{x},[\widehat{x}, \rho]]+\frac{\gamma}{2}[\widehat{x},\{\widehat{p}, \rho\}]-i \Omega r^{2}[\widehat{p},[\widehat{p}, \rho]]
$$

where $\Lambda, \gamma$ and $\Omega$ are real time-dependent coefficients. As discussed in Appendix B (80) a possible model for $\Omega(t)$ is

$$
\Omega(t)=\Omega_{0}+\frac{\widetilde{\gamma}}{a+t}+\frac{\widetilde{\Gamma}}{1+b t^{2}}
$$

where $\Omega_{0}, \widetilde{\gamma}, a, \widetilde{\Gamma}$ and $b$ are positive constants, including appropriate powers of $\alpha^{\prime}$. The quantity $\widetilde{\gamma}<1$ contains information on the density of D-particle defects on a fourdimensional world. From the earlier derivation of the master equation it is clear that the last term of the r.h.s. of (18) represents a time-dependent cosmological constant contribution. The form of this time dependence, just as other issues to do with the cosmological constant, is a matter of debate. Arguments, for example, arising from quintessence [29] and cosmon scalar fields lead to a behaviour which is compatible with (18). Similar behaviour is also obtained in dark.energy relaxation models in the context of non-critical strings (linear-dilaton models [30, 31] or colliding-brane worlds (recoil) [22, 12]). The time dependence of $\gamma$ and $\Lambda$ can be calculated in the weak coupling limit for general $n$ (i.e. ohmic, $n=1$ and non-ohmic $n \neq 1$ environments) where

$$
I(\omega)=\frac{2}{\pi} m \gamma_{0} \omega\left[\frac{\omega}{\varpi}\right]^{n-1} e^{-\omega^{2} / \omega^{2}}
$$

and $\varpi$ is a cut-off frequency. The precise time dependence is governed by $\Lambda(t)=\int_{0}^{t} d s \nu(s)$ and $\gamma(t)=\int_{0}^{t} d s \nu(s) s$ where $\nu(s)=\int_{0}^{\infty} d \omega I(\omega) \operatorname{coth}(\beta \hbar \omega / 2) \cos (\omega s)$. For the ohmic case, in the limit $\hbar \varpi \ll k_{B} T$ followed by $\varpi \rightarrow \infty, \Lambda$ and $\gamma$ are given by $m \gamma_{0} k_{B} T$ and $\gamma_{0}$ respectively after a rapid initial transient. For high temperatures $\Lambda$ and $\gamma$ have a powerlaw increase with $t$ for the subohmic case whereas there is a rapid decrease in the supraohmic case. However for our considerations it is adequate to restrict attention to the ohmic high temperature limit [32].

\section{Solution of the master equation}

The master equation of (17) can be solved. It is useful to introduce the operator

$$
\widehat{D}=\exp (i(k \widehat{x}+\Delta \widehat{p}))
$$


and the transform

$$
\widetilde{\rho}(\kappa, \Delta)=\operatorname{tr}(\rho \exp (i(\kappa \widehat{x}+\Delta \widehat{p}))) .
$$

The master equations then takes the form

$$
\frac{\partial}{\partial t} \widetilde{\rho}+\left(-\frac{\kappa}{m}+\gamma \Delta\right) \frac{\partial}{\partial \Delta} \widetilde{\rho}=-\Lambda \Delta^{2} \widetilde{\rho}-\Omega(t) r^{2} \kappa^{2} \widetilde{\rho} .
$$

This is solved by [33]

$$
\widetilde{\rho}(\kappa, \Delta, t)=\digamma\left(e^{-\gamma t}\left(\Delta-\frac{\kappa}{m \gamma}\right), \kappa\right) \mathcal{P}_{I}(\kappa, \Delta, t)
$$

where $\mathcal{P}_{I}$ is a particular solution of 22 and $\digamma\left(e^{-\gamma t}\left(\Delta-\frac{\kappa}{m \gamma}\right), \kappa\right)$ is an arbitrary solution of

$$
\frac{\partial}{\partial t} \widetilde{\rho}+\left(-\frac{\kappa}{m}+\gamma \Delta\right) \frac{\partial}{\partial \Delta} \widetilde{\rho}=0
$$

for an arbitrary $\digamma$.

We can write

$$
\mathcal{P}_{I}(\kappa, \Delta, t)=\exp \left(f_{1}(t) \kappa^{2}+f_{2}(t) \Delta^{2}+f_{3}(t) \kappa \Delta\right) .
$$

It is then necessary that

$$
\begin{aligned}
\frac{d}{d t} f_{1}-\frac{f_{3}}{m} & =-r^{2} \Omega(t) \\
\frac{d}{d t} f_{2}+2 \gamma f_{2} & =-\Lambda \\
\frac{d}{d t} f_{3}-\frac{2}{m} f_{2}+\gamma f_{3} & =0 .
\end{aligned}
$$

Now (26) is a stochastic differential equation.We can consider $r(t)$ to be a (finite variance) Gaussian variable with variance $\sigma^{2}$ and vanishing mean. In the ohmic high temperature limit and in the mean we can solve these coupled equations, for initial conditions $f_{1}(0)=$ $f_{2}(0)=f_{3}(0)=0$ to give

$$
\begin{aligned}
f_{1}(t)= & \frac{1}{2 m \gamma^{2} \sqrt{b}}\left(k_{B} T \sqrt{b}\left(-2 \gamma t-\left(1-e^{-2 \gamma t}\right)+4\left(1-e^{-\gamma t}\right)\right)-\right. \\
& \left.2 m \sigma^{2} \gamma^{2} \widetilde{\Gamma} \tan ^{-1}(\sqrt{b} t)\right) \\
f_{2}(t)= & \frac{1}{2} m k_{B} T\left(e^{-2 \gamma t}-1\right) \\
f_{3}(t)= & \frac{k_{B} T}{\gamma}\left(2\left(e^{-\gamma t}-1\right)+\left(1-e^{-2 \gamma t}\right)\right)
\end{aligned}
$$


on taking $\Omega_{0}=\widetilde{\gamma}=0$. In order to study interference we need to understand the behaviour of $\rho\left(x, x^{\prime}\right) \cdot \rho$ and $\widetilde{\rho}$ are related by

$$
\rho\left(x, x^{\prime}, t\right)=\frac{1}{2 \pi} \int_{-\infty}^{\infty} d \kappa e^{-i \kappa\left(x+x^{\prime}\right) / 2} \widetilde{\rho}\left(\kappa, x-x^{\prime}, t\right) .
$$

For an initial state which is a linear superposition $\left|k_{1}\right\rangle+\left|k_{2}\right\rangle$ of momentum eigenstates

$\widetilde{\rho}(\kappa, \Delta, t=0)=\pi e^{i \kappa \Delta / 2}\left(\delta(\kappa)\left(e^{i \Delta k_{1}}+e^{i \Delta k_{2}}\right)+\delta\left(\kappa+k_{2}-k_{1}\right) e^{i \Delta k_{2}}+\delta\left(\kappa+k_{1}-k_{2}\right) e^{i \Delta k_{1}}\right)$

and so

$\digamma(\Delta, \kappa)=\pi e^{i \frac{\kappa}{2}\left(\Delta+\frac{\kappa}{m \gamma}\right)}\left(\begin{array}{c}\delta(\kappa)\left(e^{i\left(\Delta+\frac{\kappa}{m \gamma}\right) k_{1}}+e^{i\left(\Delta+\frac{\kappa}{m \gamma}\right) k_{2}}\right)+\delta\left(\kappa+k_{2}-k_{1}\right) e^{i\left(\Delta+\frac{\kappa}{m \gamma}\right) k_{2}} \\ +\delta\left(\kappa+k_{1}-k_{2}\right) e^{i\left(\Delta+\frac{\kappa}{m \gamma}\right) k_{1}}\end{array}\right)$.

This implies that

$$
\rho(x, x-\Delta, t)=\frac{1}{2}\left(\begin{array}{c}
e^{f_{2}(t) \Delta^{2}+i k_{1} g(t, 0, \Delta)}+e^{f_{2}(t) \Delta^{2}+i k_{2} g(t, 0, \Delta)}+ \\
e^{f_{1}(t)\left(k_{1}-k_{2}\right)^{2}+f_{2}(t) \Delta^{2}+f_{3}(t)\left(k_{1}-k_{2}\right) \Delta} e^{i\left[\left(k_{1}-k_{2}\right)\left(\frac{\Delta}{2}-x\right)+\frac{\left(k_{1}+k_{2}\right)}{2} g\left(t, k_{1}-k_{2}, \Delta\right)\right]}+ \\
e^{f_{1}(t)\left(k_{2}-k_{1}\right)^{2}+f_{2}(t) \Delta^{2}+f_{3}(t)\left(k_{2}-k_{1}\right) \Delta} e^{i\left[-\left(k_{1}-k_{2}\right)\left(\frac{\Delta}{2}-x\right)+\frac{\left(k_{1}+k_{2}\right)}{2} g\left(t, k_{2}-k_{1}, \Delta\right)\right]}
\end{array}\right)
$$

where $g(t, \kappa, \Delta)=e^{-\gamma t}\left(\Delta-\frac{\kappa}{m \gamma}\right)+\frac{\kappa}{m \gamma}$.

The form of interference manifests itself in $\rho(x, x, t)$ :

$$
\rho(x, x, t)=1+\exp \left(f_{1}(t)\left(k_{1}-k_{2}\right)^{2}\right)\left\{\cos \left(\left[k_{1}-k_{2}\right] x-\frac{\left(k_{1}^{2}-k_{2}^{2}\right)}{2 m \gamma}\left(1-e^{-\gamma t}\right)\right)\right\}
$$

Clearly from the form of $f_{1}(t)(29)$ the above indicates that the reduction in contrast is not exponential with $t$; hence the interference is qualitatively of a milder form than that due to conventional baths which includes the approach due to quantum semi-groups.

\section{Flavour oscillation}

In order to understand the effect of decoherence on flavour oscillations we can for simplicity consider two flavours and flavours created with a sharp momentum. Consequently for any momentum ket $|k\rangle$ there will be two possible quantum numbers corresponding to the two mass eigenstates with mass eigenvalues $m_{1}$ and $m_{2}$. To reflect this the density matrix will have a $2 \times 2$ matrix structure

$$
\rho=\rho_{0} \mathbf{1}+\rho_{i} \sigma_{i}
$$


where $\sigma_{i}, i=1 \ldots 3$ are the Pauli matrices and $\mathbf{1}$ is the identity matrix and a summation convention over repeated indices is assumed. In terms of

$$
m_{12}=\frac{m_{2}-m_{1}}{m_{2}+m_{1}}, \frac{1}{m}=\frac{1}{2}\left(\frac{1}{m_{1}}+\frac{1}{m_{2}}\right)
$$

we can write the kinetic energy as $H_{0}=\frac{\widehat{p}^{2}}{2 m}\left(\mathbf{1}+m_{12} \sigma_{3}\right)$. Because the decoherence is gravitational it will be sensitive to masses; hence we can take it to have in general a structure with $r$ no longer a scalar function. A random $2 \times 2$ matrix structure $r=r_{0} \mathbf{1}+r_{i} \sigma_{i}$ would be natural. The previous master equation generalises to

$$
i \frac{d}{d t}\left(\rho_{0} \mathbf{1}+\rho_{j} \sigma_{j}\right)=\left[H_{0}, \rho_{0} \mathbf{1}+\rho_{j} \sigma_{j}\right]-i \Omega\left[\widehat{p}\left(r_{0} \mathbf{1}+r_{i} \sigma_{i}\right),\left[\widehat{p}\left(r_{0} \mathbf{1}+r_{j} \sigma_{j}\right), \rho_{0} \mathbf{1}+\rho_{k} \sigma_{k}\right]\right]
$$

We now introduce

$$
\widetilde{\rho}_{\mu}(\kappa, \Delta)=\operatorname{tr}\left(\rho_{\mu} \widehat{D}\right)
$$

for $\mu=0,1,2,3$.

The equations arising for $\rho_{\mu}$ are given in the appendix. In order to draw qualitative conclusions it is sufficient to simplify by requiring only $r_{0}$ and $r_{3}$ as being the only non-zero random variables. The analysis then simplifies and is given in Appendix A.

The flavour mixing unitary matrix is

$$
U=\left(\begin{array}{cc}
\cos \theta & \sin \theta \\
-\sin \theta & \cos \theta
\end{array}\right)
$$

and relates flavour to mass eigenstates through $|\alpha\rangle=U_{\alpha j}|j\rangle$ ( where the Latin indices apply to mass eigenstates and Greek indices to flavour eigenstates). The initial density matrix will represent a particle in flavour eigenstate 1 with momentum $p$. Its matrix elements are

$$
\begin{aligned}
& \rho_{11}(t=0)=\cos ^{2} \theta|p\rangle\langle p| \\
& \rho_{22}(t=0)=\sin ^{2} \theta|p\rangle\langle p| \\
& \rho_{12}(t=0)=\sin \theta \cos \theta|p\rangle\langle p| \\
& \rho_{21}(t=0)=\sin \theta \cos \theta|p\rangle\langle p| .
\end{aligned}
$$

The corresponding elements for $\widetilde{\rho}$ are

$$
\begin{aligned}
& \widetilde{\rho}_{0}(t=0)=\pi e^{i \Delta(p+\kappa / 2)} \delta(\kappa) \\
& \widetilde{\rho}_{3}(t=0)=\pi \cos (2 \theta) e^{i \Delta(p+\kappa / 2)} \delta(\kappa) \\
& \widetilde{\rho}_{1}(t=0)=\pi \sin (2 \theta) e^{i \Delta(p+\kappa / 2)} \delta(\kappa) \\
& \widetilde{\rho}_{2}(t=0)=0
\end{aligned}
$$


The solution at a general time (c.f. Appendix A) is

$$
\begin{aligned}
& \widetilde{\rho}_{0}(t)=\frac{\pi}{2} e^{i \Delta(p+\kappa / 2)} \delta(\kappa)\left\{\begin{array}{c}
(1+\cos (2 \theta)) \exp \left[-t\left(\left(r_{0}+r_{3}\right)^{2} \kappa^{2} \frac{\mathcal{I}(t)}{t}-i \frac{\left(1+m_{12}\right) \kappa(2 p+\kappa)}{2 m}\right)\right]+ \\
(1-\cos (2 \theta)) \exp \left[-t\left(\left(r_{0}-r_{3}\right)^{2} \kappa^{2} \frac{\mathcal{I}(t)}{t}-i \frac{\left(1-m_{12}\right) \kappa(2 p+\kappa)}{2 m}\right)\right]
\end{array}\right\} \\
& \widetilde{\rho}_{3}(t)=\frac{\pi}{2} e^{i \Delta(p+\kappa / 2)} \delta(\kappa)\left\{\begin{array}{c}
(1+\cos (2 \theta)) \exp \left[-t\left(\left(r_{0}+r_{3}\right)^{2} \kappa^{2} \frac{\mathcal{I}(t)}{t}-i \frac{\left(1+m_{12}\right) \kappa(2 p+\kappa)}{2 m}\right)\right]- \\
(1-\cos (2 \theta)) \exp \left[-t\left(\left(r_{0}-r_{3}\right)^{2} \kappa^{2} \frac{\mathcal{I}(t)}{t}-i \frac{\left(1-m_{12}\right) \kappa(2 p+\kappa)}{2 m}\right)\right]
\end{array}\right\} \\
& \widetilde{\rho}_{1}(t)=\pi \sin (2 \theta) e^{i \Delta(p+\kappa / 2)} \delta(\kappa) \cos \left(\frac{m_{12}}{m} p^{2} t\right) \exp \left(-4 r_{3}^{2} p^{2} \mathcal{I}(t)\right) \\
& \widetilde{\rho}_{2}(t)=\pi \sin (2 \theta) e^{i \Delta(p+\kappa / 2)} \delta(\kappa) \sin \left(\frac{m_{12}}{m} p^{2} t\right) \exp \left(-4 r_{3}^{2} p^{2} \mathcal{I}(t)\right)
\end{aligned}
$$

where (c.f. (62)):

$$
\mathcal{I}(t) \equiv \int_{0}^{t} \Omega\left(t^{\prime}\right) d t^{\prime}=\Omega_{0} t+\widetilde{\gamma} \ln (1+t / a)+\frac{\widetilde{\Gamma}}{\sqrt{b}} \tan ^{-1}(\sqrt{b} t)
$$

The probability for the transition of flavour 1 to flavour 2 at time $t$ is $\operatorname{tr}\left(\rho_{f \mu} \rho_{\mu}(t)\right)$ where

$$
\rho_{f 0}=\frac{1}{2}|p\rangle\left\langle p\left|, \rho_{f 1}=-\frac{1}{2} \sin 2 \theta\right| p\right\rangle\left\langle p\left|, \rho_{f 2}=0, \rho_{f 3}=-\frac{1}{2} \cos 2 \theta\right| \mathrm{p}\right\rangle\langle\mathrm{p}| .
$$

From (43), (44) it is clear that decoherence affects this probability with an exponential damping only if the cosmological term $\Omega$ is the constant $\Omega_{0}$. In particular, in the absence of the $\Omega_{0}$ term, and in the limit $\widetilde{\Gamma}=0$, the decoherence due to the D-particle foam results in power damping for large times $t \rightarrow \infty$, the terms $\widetilde{\rho}_{i} \sim t^{-\delta_{i}^{2}}, i=0,3$, while $\widetilde{\rho}_{i} \sim t^{-p^{2} \delta_{i}^{2}}$, $i=1,2$, i.e. the scaling power depends on the probe's momentum (with $\delta_{i}$ appropriate constants depending on the term we look at in (44). With the general $\Omega(t)$ of (18) the probability for flavour oscillation, $P_{1 \rightarrow 2}$, is proportional to

$$
P_{1 \rightarrow 2} \propto(\sin 2 \theta)^{2}\left(1-\exp \left[-4 \sigma^{2} p^{2} \mathcal{I}(t)\right]\right) \cos \left(\frac{m_{12} p^{2} t}{m}\right) .
$$

with $(t)$ given by (44).

We remark at this stage that, in view of the fundamental CPT violation inherent in the master equation (13) or (17), as a result of the microscopic time irreversibility under $t \rightarrow-t$ of the entanglement terms of this equation, it is also possible (but not necessary) to consider models of D-foam in which the momentum transfer (15), (16) differs between particle and antiparticle sectors. In such a case, one would then obtain different decoherence coefficients for particles and antiparticles, a model which was already used in [17] in order to fit LSND data [16].

Although our simplified model for flavour oscillations, using a non-relativistic bosonic two-level system, is too simplified for realistic phenomenology of (neutrino) flavour oscillations, nevertheless we believe that it captures the basic features induced by Liouvilledecoherence, which are expected to persist in the relativistic neutrino case. The important 
point is the damping factor $\exp \left[-4 \sigma^{2} p^{2} \mathcal{I}(t)\right]$ in front of the oscillatory term, which depends on the square of the momentum of the probe, as well as the space-time foam characteristics, such as the dispersion $\sigma^{2}$ (16) in the momentum transfer during the interaction of the matter probe with the D-particle defect.

In this respect, it is interesting to compare the general form (45) of the Liouville decoherence, in which the entanglement is time dependent in general, with the generic models of Lindblad decoherence, based on the approach of [2, in which the environmental entanglement is characterized by a constant decoherence matrix. For instance, consider for definiteness the two-flavour oscillation case, in the completely-positive neutrino decoherence model of [34, 35], for which there is only one real and positive, constant in time, but possibly probe-energy dependent, decoherence parameter $\gamma$ to characterize the quantumgravity entanglement. In the case where the energy and lepton number of the neutrino are assumed conserved (on average, at least) in the presence of quantum-gravity fluctuations, the oscillation probability reads in that model:

$$
P_{\nu_{\alpha} \rightarrow \nu_{\beta}}=\frac{1}{2}(\sin 2 \theta)^{2}\left(1-\exp (-\gamma L) \cos \left(\frac{\left(m_{1}^{2}-m_{2}^{2}\right) L}{2 E_{\nu}}\right)\right)
$$

where $L=t$ is the neutrino oscillation length, and $E_{\nu}$ is the average energy of the neutrino beam.

First of all let us concentrate in the form of the oscillatory terms. To understand the difference between our case (45) and (46), we should remind the reader that flavour oscillations can be analyzed in two ways: the first involves flavours with sharp momentum, in which case one considers oscillations in time, as done in the present work, whilst the other involves flavour with sharp energy, in which case the oscillations are in space. This is the conventional neutrino case, leading to (46). The conclusions should not change, however, qualitatively. For our purposes it is not necessary, therefore, to consider the second case in order to get a qualitative description of the D-foam effects. This will be important only when we embark on a detailed phenomenology, by looking at specific experiments, in which case complications involving wave packets and the joint presence of the above effects must be incorporated. One should also take into account that the neutrino is a highly relativistic system, with a very small mass, in contrast to our non-relativistic bosonic system examined in this section for simplicity. One expects, therefore, that when we consider in our approach relativistic systems, with sharp energy, a similar form for the oscillatory term as in (46) will be obtained. Qualitatively, however, in both (45) and (46), the main reason for oscillations is the conventional mass difference between the mass eigenstates, which agrees with the present phenomenology.

The exponential damping (with time or oscillation length) induced by the decoherence term is only present in our case in the case of a cosmological constant. The generic D-foam effects are time dependent in the way indicated in (44), which does not introduce exponential damping. The important feature, however, is the quadratic momentum dependence $p^{2}$ of the exponent of these "damping" terms, which persists in the relativistic case, since it originates from the specific $[p,[p, \rho]]$ diffusion term of the master equation (17), as well as their dependence on the specific characteristics of the foam, such as the dispersion $\sigma$ 
(16) for the momentum transfer during the interaction of the particle probe with the foam. Experimentally, therefore, one should be able to bound this dispersion by comparing the oscillation probability (45) as a function of the oscillation length (time) with the corresponding experimental curve. Currently there are stringent limits from neutrino physics on quantum decoherence terms [36, and one can envisage that such bounds provide important restrictions on our D-particle foam characteristic dispersion $\sigma$, when the model is applied to relativistic neutrino beams. We postpone a detailed phenomenological analysis of our effects for a future publication, due to the complications mentioned above.

It is also worth noticing at this stage that damping factors, which resemble those due to decoherence, can arise simply 37] by conventional uncertainties in the energy (or momentum, depending of the formalism adopted) of the neutrino beam. If $\sigma_{E}$ denotes the corresponding dispersion, then, for the two-flavour oscillation problem one finds:

$$
P_{\nu_{\alpha} \rightarrow \nu_{\beta}}=\frac{1}{2}(\sin 2 \theta)^{2}\left(1-\exp \left(-2 \sigma_{E}^{2}\left(m_{1}^{2}-m_{2}^{2}\right)^{2}\right) \cos \left(\frac{\left(m_{1}^{2}-m_{2}^{2}\right)\langle L\rangle}{2\left\langle E_{\nu}\right\rangle}\right)\right)
$$

The reader is invited to compare (47) with (45) and (46). We note that by writing [37): $\sigma_{E}=\frac{L}{4 E_{\nu}} r$, with $r \sim \delta E / E$ (ignoring, as negligible, uncertainties in oscillation lengths), then one may rewrite (47) in a decoherence form (46) with the decoherence coefficient $\gamma \sim$ $\left(m_{1}^{2}-m_{2}^{2}\right)^{2} L r^{2} / 8 E_{\nu}^{2}$. For atmospheric neutrinos, this yields the bound $\gamma_{\mathrm{atm}} \leq 10^{-24} \mathrm{GeV}$, assuming $r^{2}=(1)$. Similar bounds can be obtained for the D-foam decoherence damping factor (45), which notably is independent of the mass difference. Indeed, one can also formally rewrite the damping factor in (45) as a decoherence (46) term, with $\gamma=4 \sigma^{2} p^{2} I(L) / L$, with $L=t$. However, for reasons explained above this formula is not quite precise, and for quantitative comparison one should repeat the analysis with relativistic neutrino systems, in the sharp energy formalism. This is left for future work.

Finally, before closing this section we would like to compare our result (45) with the energy-driven Lindblad decoherence models for two-flavour oscillations considered in 38]. In the latter case, the pertinent master equation for the density matrix of matter propagating in a self-adjoint Lindblad environment, spanned by operators $D_{n}^{\dagger}=D_{n}$, with $\left[D_{n}, H\right]=0$, reads (in units $\hbar=1=c$ ):

$$
\partial_{t} \rho=i[\rho, H]-\sum_{n}\left[D_{n},\left[D_{n}, \rho\right]\right]
$$

When specialized to a two-level system, Adler [38] asserts that the only possible choices that commute with $H$ are $D_{n}=\kappa_{n} \widehat{1}$, with $\widehat{1}$ the identity operator, and $D_{n}=\lambda_{n} H$, with $\kappa_{n}, \lambda_{n}$ appropriate constants. In the relativistic massive neutrino case, with fixed energy $E$ for the neutrino beam, $H \simeq p+\frac{m^{2}}{2 p}$, from which, by projecting onto mass eigenstates, and concentrating on the non-diagonal density-matrix elements, Eq. (48) yields the following leading-order estimate for the decoherence coefficient $\gamma[38$ :

$$
\gamma \sim \frac{\left(m_{1}^{2}-m_{2}^{2}\right)^{2}}{4 E^{2} M_{P}}
$$


where $1 / M_{P}=\sum_{n} \lambda_{n}^{2}$ is a characteristic Quantum Gravity scale. In the non-relativistic

case at hand, from the rest-mass terms in the expansion of the Hamiltonian $H \simeq m+\frac{p^{2}}{2 m}$, we obtain the following estimate for the decoherence term $\gamma_{n r} \sim\left(m_{1}-m_{2}\right)^{2} / M_{P}+\mathcal{O}\left(p^{4}\left(m_{1}-\right.\right.$ $\left.\left.m_{2}\right)^{2} / 4 m_{1}^{2} m_{2}^{2} M_{P}\right)$. These estimates are much more suppressed.than the estimates of [34], who allowed for the decoherence coefficients (in the relativistic case) to be of order $E^{2} / M_{P}$, with $E$ a fixed neutrino energy.

We now note that this latter, quadratic in energy, dependence of the decoherence coefficient appears to be the case of our Liouville decoherence, and owes its form to the peculiar (quadratic) momentum dependence of the Liouville environmental decoherening entanglement term of the master equation (17). The form of this term is independent of the relativistic or non-relativistic nature of the Hamiltonian $H$, whilst the form of (48), (49) depends crucially on the form of the Hamiltonian $H$.

In the Liouville decoherence case of the D-particle foam, examined in the present work, the Lindblads, given by $\bar{u} \propto r \widehat{p}$ (c.f. (15)), although self-adjoint and commuting with the Hamiltonian of the non-relativistic system, nevertheless are not proportional to it. This is due to the fact that the Lindblad operators in this case are proportional to the metric tensor (13), as a result of the underlying general coordinate invariance of the Liouvillestring system, which is not taken into account in the simple quantum mechanical case of 38. This leads to an entirely different energy (momentum) dependence $\left(\propto p^{2}\right)$ of the decoherence coefficient in the Liouville case, as we have seen above. With these comments we conclude our phenomenological analysis on Liouville decoherence.

\section{Conclusions}

In this work we have examined phenomenological consequences of a decoherence master equation describing the propagation of low-energy string matter in a space-time foam background. The basic machinery was the Liouville-string approach to decoherence.

We have restricted ourselves in a specific model for the space time foam, involving the interaction of strings with space-time D-particle defects. We have seen that the pertinent master equation exhibits diffusion terms and decoherence, but that there is not always exponential damping. The reason is the time dependence of some of the decoherence terms. The Liouville decoherence involves a term which is quadratic in the momentum transfer encountered during the interaction of the particle probe with the space-time defect. By considering a specific model of random foam, characterized by the dispersion of such a momentum transfer, we have been able to solve the pertinent decoherence equation rather simply, and give the form of the corresponding probability for the oscillation from one flavour to another, in a toy non-relativistic bosonic model of two flavour oscillations. Our formalism also allows for the consideration of possible decoherence effects arising from dark energy contributions in the space time. Such effects have also been taken into account in the expression for the final oscillation probability

Although the model is not strictly appropriate for neutrino physics, nevertheless it exhibits the basic properties of Liouville decoherence that one expects to persist in the fully 
relativistic neutrino case. The basic feature is a damping looking factor $\exp \left(-4 p^{2} \sigma^{2} \mathcal{I}(t)\right)$, in front of the conventional oscillation terms that are due to the mass difference of the pertinent mass eigenstates. The damping factor depends on the characteristics of the foam, but leads to exponential damping (with the time $t$ ) only in case there is a cosmological constant term in space time.

The simplified analysis presented in the current article demonstrates that generic phenomenological analyses, based on constant decoherence coefficients, might be misleading, yielding sometimes incorrect bounds of the relevant space-time foam effects. One should resort, whenever possible, to rather detailed microscopic models of space-time foam, as we have done here, before embarking on detailed phenomenological analyses. One should also be careful to disentangle conventional matter effects, or effects related simply to uncertainties in the energy or oscillation lengths, before concluding on the possible rôle of fundamental physics effects in particle processes, such as flavour oscillation. We hope to return to a detailed phenomenological analysis of D-particle foam effects in neutrinos and other particle probes, taking into account of the appropriate spin structures as well, in a future publication.

\section{Acknowledgements}

We acknowledge discussions with G. Barenboim and A. Waldron-Lauda.

\section{Appendix A: Detailed solution of the master equation}

By multiplying (39) with $\sigma_{\mu}$ and taking traces the following equations arise:

$$
\frac{\partial}{\partial t} \widetilde{\rho}_{0}=\frac{\kappa}{m}\left(\frac{\partial}{\partial \Delta} \widetilde{\rho}_{0}+m_{12} \frac{\partial}{\partial \Delta} \widetilde{\rho}_{3}\right)-\Omega \kappa^{2}\left(r_{\mu} r_{\mu} \widetilde{\rho}_{0}+2 r_{0} r_{j} \widetilde{\rho}_{j}\right)
$$

and

$$
\begin{aligned}
\frac{\partial}{\partial t} \widetilde{\rho}_{j}= & \left(\frac{m_{12}}{m} \varepsilon_{3 l j}+4 \Omega h_{l j}\right)\left(i \frac{\partial}{\partial \Delta}+\frac{\kappa}{2}\right)^{2} \widetilde{\rho}_{l}-4 i \Omega \kappa f_{l j}\left(i \frac{\partial}{\partial \Delta}+\frac{\kappa}{2}\right) \widetilde{\rho}_{l}+ \\
& \frac{\kappa}{m}\left(m_{12} \delta_{j 3} \frac{\partial}{\partial \Delta} \widetilde{\rho}_{0}+\left(\delta_{i j}-i m_{12} \varepsilon_{3 l j}\right) \frac{\partial}{\partial \Delta} \widetilde{\rho}_{l}\right)- \\
& \Omega \kappa^{2}\left(2 r_{0} r_{j} \widetilde{\rho}_{0}+\left(r_{l} r_{j}+r_{0}^{2} \delta_{j l}+i r_{0} \varepsilon_{l k j} r_{k}-i f_{l j}\right) \widetilde{\rho}_{l}\right)
\end{aligned}
$$

where $f_{j p}=r_{0} r_{s} \varepsilon_{j p s}+i r_{l} r_{l} \delta_{j p}-i r_{j} r_{p}$.

When $r_{0}$ and $r_{3}$ are the only non-zero components of $r_{\mu}$ then the above equations 
become

$$
\begin{aligned}
\frac{\partial}{\partial t} \widetilde{\rho}_{0}= & \frac{\kappa}{m}\left(\frac{\partial}{\partial \Delta} \widetilde{\rho}_{0}+m_{12} \frac{\partial}{\partial \Delta} \widetilde{\rho}_{3}\right)-\Omega \kappa^{2}\left(\left(r_{0}^{2}+r_{3}^{2}\right) \widetilde{\rho}_{0}+2 r_{0} r_{3} \widetilde{\rho}_{3}\right) \\
\frac{\partial}{\partial t} \widetilde{\rho}_{1}= & -\left(i \frac{\partial}{\partial \Delta}+\frac{\kappa}{2}\right)^{2}\left(\frac{m_{12}}{m} \widetilde{\rho}_{2}+4 \Omega r_{3}^{2} \widetilde{\rho}_{1}\right)-4 i \Omega \kappa\left(i \frac{\partial}{\partial \Delta}+\frac{\kappa}{2}\right)\left(-r_{0} r_{3} \widetilde{\rho}_{2}+i r_{3}^{2} \widetilde{\rho}_{1}\right)+ \\
& \frac{\kappa}{m}\left(\frac{\partial}{\partial \Delta} \widetilde{\rho}_{1}+i m_{12} \frac{\partial}{\partial \Delta} \widetilde{\rho}_{2}\right)-\Omega \kappa^{2}\left(\left(r_{0}^{2}+r_{3}^{2}\right) \widetilde{\rho}_{1}+2 i r_{0} r_{3} \widetilde{\rho}_{2}\right) \\
\frac{\partial}{\partial t} \widetilde{\rho}_{2}= & \left(i \frac{\partial}{\partial \Delta}+\frac{\kappa}{2}\right)^{2}\left(\frac{m_{12}}{m} \widetilde{\rho}_{1}-4 \Omega r_{3}^{2} \widetilde{\rho}_{2}\right)-4 i \Omega \kappa\left(i \frac{\partial}{\partial \Delta}+\frac{\kappa}{2}\right)\left(r_{0} r_{3} \widetilde{\rho}_{1}+i r_{3}^{2} \widetilde{\rho}_{2}\right)+ \\
& \frac{\kappa}{m}\left(\frac{\partial}{\partial \Delta} \widetilde{\rho}_{2}-i m_{12} \frac{\partial}{\partial \Delta} \widetilde{\rho}_{1}\right)-\Omega \kappa^{2}\left(\left(r_{0}^{2}+r_{3}^{2}\right) \widetilde{\rho}_{2}-2 i r_{0} r_{3} \widetilde{\rho}_{1}\right) \\
\frac{\partial}{\partial t} \widetilde{\rho}_{3}= & \frac{\kappa}{m}\left(\frac{\partial}{\partial \Delta} \widetilde{\rho}_{3}+m_{12} \frac{\partial}{\partial \Delta} \widetilde{\rho}_{0}\right)-\Omega \kappa^{2}\left(2 r_{0} r_{3} \widetilde{\rho}_{0}+\left(r_{0}^{2}+r_{3}^{2}\right) \widetilde{\rho}_{3}\right)
\end{aligned}
$$

The equations (52), (153), (54) and (55) form two decoupled sets, one involving $\widetilde{\rho}_{0}$ and $\widetilde{\rho}_{3}$, the other $\widetilde{\rho}_{1}$ and $\widetilde{\rho}_{2}$. In order to solve these equations we introduce the Fourier transforms $\widetilde{\widetilde{\rho}}_{\mu}$ defined by

$$
\widetilde{\widetilde{\rho}}_{\mu}(\kappa, \eta)=\int_{-\infty}^{\infty} \widetilde{\rho}_{\mu}(\kappa, \Delta) e^{-2 \pi i \eta \Delta} d \Delta
$$

(so that $\left.\widetilde{\rho}_{\mu}(\kappa, \Delta)=\int_{-\infty}^{\infty} \widetilde{\widetilde{\rho}}_{\mu}(\kappa, \eta) e^{2 \pi i \eta \Delta} d \eta\right)$.

We then have

$$
i \frac{\partial}{\partial t}\left(\begin{array}{c}
\widetilde{\widetilde{\rho}}_{0} \\
\widetilde{\widetilde{\rho}}_{3}
\end{array}\right)=M\left(\begin{array}{l}
\widetilde{\widetilde{\rho}}_{0} \\
\widetilde{\widetilde{\rho}}_{3}
\end{array}\right)
$$

where

$$
M=\left(\begin{array}{cc}
-\frac{2 \pi \kappa \eta}{m}-i \Omega \kappa^{2}\left(r_{o}^{2}+r_{3}^{2}\right) & -\frac{2 \pi \kappa \eta m_{12}}{m}-2 i \Omega \kappa^{2} r_{0} r_{3} \\
-\frac{2 \pi \kappa \eta m_{12}}{m}-2 i \Omega \kappa^{2} r_{0} r_{3} & -\frac{2 \pi \kappa \eta}{m}-i \Omega \kappa^{2}\left(r_{o}^{2}+r_{3}^{2}\right)
\end{array}\right) .
$$

Now for $\mathcal{C}=\left(\begin{array}{cc}1 & -1 \\ 1 & 1\end{array}\right)$

$$
\mathcal{C}^{-1} M \mathcal{C}=\left(\begin{array}{cc}
\lambda_{1} & 0 \\
0 & \lambda_{2}
\end{array}\right)
$$

where

$$
\begin{aligned}
& \lambda_{1}=-\frac{\kappa}{m}\left(2\left(1+m_{12}\right) \pi \eta+i m\left(r_{0}+r_{3}\right)^{2} \kappa \Omega\right) \\
& \lambda_{2}=-\frac{\kappa}{m}\left(2\left(1-m_{12}\right) \pi \eta+i m\left(r_{0}-r_{3}\right)^{2} \kappa \Omega\right) .
\end{aligned}
$$

Notice that so far $\Omega$ has been kept arbitrary, in other words it may be in general a function of (cosmic) time $\Omega(t)$. 
On introducing

$$
\left(\begin{array}{c}
\widehat{\rho}_{0} \\
\widehat{\rho}_{3}
\end{array}\right)=\mathcal{C}^{-1}\left(\begin{array}{c}
\widetilde{\widetilde{\rho}}_{0} \\
\widetilde{\widetilde{\rho}}_{3}
\end{array}\right)
$$

we have

$$
\begin{aligned}
& \widehat{\rho}_{0}(t)=e^{-i \int_{0}^{t} \lambda_{1}\left(t^{\prime}\right) d t^{\prime}} \widehat{\rho}_{0}(0) \\
& \widehat{\rho}_{3}(t)=e^{-i \int_{0}^{t} \lambda_{2}\left(t^{\prime}\right) d t^{\prime}} \widehat{\rho}_{3}(0) .
\end{aligned}
$$

Similarly

$$
i \frac{\partial}{\partial t}\left(\begin{array}{c}
\widetilde{\widetilde{\rho}}_{1} \\
\widetilde{\widetilde{\rho}}_{2}
\end{array}\right)=N\left(\begin{array}{l}
\widetilde{\widetilde{\rho}}_{1} \\
\widetilde{\widetilde{\rho}}_{2}
\end{array}\right)
$$

where

$$
N_{11}=N_{22}=-\frac{2 \pi \eta \kappa}{m}-16 i \pi^{2} r_{3}^{2} \eta^{2} \Omega+i r_{3}^{2} \kappa^{2} \Omega-i\left(r_{0}^{2}+r_{3}^{2}\right) \kappa^{2} \Omega
$$

and

$$
N_{21}=-N_{12}=\frac{4 i m_{12} \pi^{2} \eta^{2}}{m}+\frac{i m_{12} \kappa^{2}}{4 m}-8 \pi r_{0} r_{3} \eta \kappa \Omega .
$$

For $\mathcal{D}=\left(\begin{array}{cc}-i & i \\ 1 & 1\end{array}\right)$

$$
\mathcal{D}^{-1} N \mathcal{D}=\left(\begin{array}{cc}
\mu_{1} & 0 \\
0 & \mu_{2}
\end{array}\right)
$$

and so, on defining

$$
\left(\begin{array}{l}
\widehat{\rho}_{1} \\
\widehat{\rho}_{2}
\end{array}\right)=\mathcal{D}^{-1}\left(\begin{array}{c}
\widetilde{\widetilde{\rho}}_{1} \\
\widetilde{\widetilde{\rho}}_{2}
\end{array}\right)
$$

we have

$$
\begin{aligned}
& \widehat{\rho}_{1}(t)=e^{-i \int_{0}^{t} \mu_{1}\left(t^{\prime}\right) d t^{\prime}} \widehat{\rho}_{1}(0) \\
& \widehat{\rho}_{2}(t)=e^{-i \int_{0}^{t} \mu_{2}\left(t^{\prime}\right) d t^{\prime}} \widehat{\rho}_{2}(0) .
\end{aligned}
$$

This analysis can be summarised as follows:

$$
\begin{aligned}
& \widetilde{\widetilde{\rho}}_{0}(t)=\frac{1}{2}\left(e^{-i \int_{0}^{t} \lambda_{1}\left(t^{\prime}\right) d t^{\prime}}+e^{-i \int_{0}^{t} \lambda_{2}\left(t^{\prime}\right) d t^{\prime}}\right) \widetilde{\widetilde{\rho}}_{0}(0)+\frac{1}{2}\left(e^{-i \int_{0}^{t} \lambda_{1}\left(t^{\prime}\right) d t^{\prime}}-e^{-i \int_{0}^{t} \lambda_{2}\left(t^{\prime}\right) d t^{\prime}}\right) \widetilde{\widetilde{\rho}}_{3}(0) \\
& \widetilde{\widetilde{\rho}}_{3}(t)=\frac{1}{2}\left(e^{-i \int_{0}^{t} \lambda_{1}\left(t^{\prime}\right) d t^{\prime}}-e^{-i \int_{0}^{t} \lambda_{2}\left(t^{\prime}\right) d t^{\prime}}\right) \widetilde{\widetilde{\rho}}_{0}(0)+\frac{1}{2}\left(e^{-i \int_{0}^{t} \lambda_{1}\left(t^{\prime}\right) d t^{\prime}}+e^{-i \int_{0}^{t} \lambda_{2}\left(t^{\prime}\right) d t^{\prime}}\right) \widetilde{\widetilde{\rho}}_{3}(0)(61) \\
& \widetilde{\widetilde{\rho}}_{1}(t)=\frac{i}{2}\left(-i\left(e^{-i \int_{0}^{t} \mu_{1}\left(t^{\prime}\right) d t^{\prime}}+e^{-i \int_{0}^{t} \mu_{2}\left(t^{\prime}\right) d t^{\prime}}\right) \widetilde{\widetilde{\rho}}_{1}(0)+\left(e^{-i \int_{0}^{t} \mu_{2}\left(t^{\prime}\right) d t^{\prime}}-e^{-i \int_{0}^{t} \mu_{1}\left(t^{\prime}\right) d t^{\prime}}\right) \widetilde{\widetilde{\rho}}_{2}(0)\right) \\
& \widetilde{\widetilde{\rho}}_{2}(t)=\frac{i}{2}\left(-\left(e^{-i \int_{0}^{t} \mu_{2}\left(t^{\prime}\right) d t^{\prime}}-e^{-i \int_{0}^{t} \mu_{1}\left(t^{\prime}\right) d t^{\prime}}\right) \widetilde{\widetilde{\rho}}_{1}(0)-i\left(e^{-i \int_{0}^{t} \mu_{1}\left(t^{\prime}\right) d t^{\prime}}+e^{-i \int_{0}^{t} \mu_{2}\left(t^{\prime}\right) d t^{\prime}}\right) \widetilde{\widetilde{\rho}}_{2}(0)\right)
\end{aligned}
$$


For the specific case where $\Omega(t)$ has the time dependence indicated in (18), the corresponding time integral is elementary:

$$
\mathcal{I}(t) \equiv \int_{0}^{t} \Omega\left(t^{\prime}\right) d t^{\prime}=\Omega_{0} t+\widetilde{\gamma} \ln (1+t / a)+\frac{\widetilde{\Gamma}}{\sqrt{b}} \tan ^{-1}(\sqrt{b} t)
$$

which, in the limit of constant $\Omega$, i.e. $\widetilde{\Gamma}, \widetilde{\gamma} \rightarrow 0$ yields the standard term $\mathcal{I}(t, \widetilde{\gamma}=0, \widetilde{\Gamma}=$ $0)=\Omega_{0} t$, responsible for the usual exponential damping. The above results are used in section 4 , where we discuss Liouville decoherence in a toy two-generation oscillation system.

\section{Appendix B: Calculation of back reaction in D-particle foam}

\section{D-particle foam contributions to master equation for Liouville- decoherence}

The material in this Appendix is a review based on [24], where we refer the reader for further details. Let us consider a $D$-particle, located at $y^{i}(t=0) \equiv y_{i}$ of the spatial coordinates of a $(d+1)$-dimensional space time (which could be a D3-brane world), which at a time $t=0$ experiences an impulse, as a result of scattering with a matter string state (see Fig. 1). In a $\sigma$-model framework, the trajectory of the $D$-particle $y^{i}(t), i=1,2, \ldots d$, a spatial index, is described by inserting the following vertex operator in the $\sigma$-model of a free string:

$$
V=\int_{\partial \Sigma} g_{i j} y^{j}(t) \partial_{n} X^{i}
$$

where $g_{i j}$ denotes the spatial components of the metric, $\partial \Sigma$ denotes the world-sheet boundary, $\partial_{n}$ is a normal world-sheet derivative, $X^{i}$ are $\sigma$-model fields obeying Dirichlet boundary conditions on the world sheet, and $t$ is a $\sigma$-model field obeying Neumann boundary conditions on the world sheet, whose zero mode is the target time. The space-time prior to Liouville dressing is assumed Euclidean for formal reasons (convergence of the corresponding $\sigma$-model path integral). We note, however, that the final Liouville-dressed target space-time acquires Minkowski signature as a result of the time-like signature of the Liouville mode [10, 22].

In the non-relativistic approximation, appropriate for a heavy D-particle defect of mass $M_{s} / g_{s}$, with $M_{s}$ the string scale, and $g_{s}$ the string coupling, assumed weak $\left(g_{s} \ll 1\right)$, the path $y^{i}(t)$ corresponding to the impulse is given by:

$$
\begin{aligned}
y_{i}(t) & =\left(\varepsilon y_{i}+u_{i} t\right) \Theta_{\varepsilon}(t) \\
u_{i} & =\left(k_{1}-k_{2}\right)_{i},
\end{aligned}
$$

with $k_{1}\left(k_{2}\right)$ the momentum of the propagating string state before (after) the recoil (see fig. 11); $y_{i}$ are the spatial collective coordinates of the D particle, and the regularized Heaviside 
functional operator $\Theta_{\varepsilon}(t)$ is given by (77) in the text [24]:

$$
\Theta_{\varepsilon}(t)=\frac{1}{2 \pi i} \int_{-\infty}^{\infty} \frac{d q}{q-i \varepsilon} e^{i q t},
$$

Eq. (64) contains actually a pair of deformations corresponding to the $\sigma$-model couplings $y_{i}$ and $u_{i}$. These deformations are relevant in a world-sheet renormalization-group sense, having anomalous scaling dimension $-\frac{\varepsilon^{2}}{2}$, i.e. to leading order in a coupling constant expansion their renormalization-group $\beta$-functions read:

$$
\beta^{y^{i}}=-\frac{\varepsilon^{2}}{2} y^{i}, \quad \beta^{u^{i}}=-\frac{\varepsilon^{2}}{2} u^{i} .
$$

The deformations form a logarithmic conformal algebra (superconformal algebra in the case of superstrings) which closes if and only if one identifies 24 the regulating parameter $\varepsilon^{-2}$ with the world-sheet renormalization-group scale $\ln |L / a|^{2}(L(a)$ is the Infrared (Ultraviolet) world-sheet scale):

$$
\varepsilon^{-2}=\eta \ln |L / a|^{2}
$$

where $\eta$ denotes the signature of time $t$ of the target-space manifold of the $\sigma$-model (prior to Liouville dressing). For Euclidean manifolds, assumed here for path-integral convergence, $\eta=+1$.

Upon the identification (67) the re-scaled couplings $\bar{y}_{i} \equiv \frac{y_{i}}{\varepsilon}$ and $\bar{u}_{i} \equiv \frac{u_{i}}{\varepsilon}$ are marginal, that is independent of the scale $\varepsilon$. It is these marginal couplings that are connected to target-space quantities of physical significance, such as the space-time back reaction of recoil, which we now proceed to calculate.

In what follows we shall concentrate only on the limit $\varepsilon \rightarrow 0$. In this limit the dominant contributions come from the $u_{i}$ recoil deformation in (64), which we shall restrict our attention to from now on. The corresponding two-point correlation function of the vertex operator associated with the deformation $u_{i}$ (Zamolodchikov metric in $u$-space $\mathcal{G}_{u u}$ ) has the leading-order behaviour [24]:

$$
\mathcal{G}_{u u} \sim \frac{1}{\varepsilon^{2}}+\text { finite terms as } \varepsilon \rightarrow 0
$$

The corresponding deformations contribute the following terms in the master equation (5):

$$
\begin{aligned}
\text { Liouville Entanglement in Eq. (5) }= & : \beta^{u_{i}} \mathcal{G}_{u_{i} u_{j}}\left[u^{j}, \rho\right]:=-\frac{\varepsilon^{2}}{2}\left[u_{i} \frac{1}{\varepsilon^{2}} \delta_{i j},\left[u^{j}, \rho\right]\right]= \\
& -\frac{1}{2}\left[u_{i},\left[u^{i}, \rho\right]\right]=-\varepsilon^{2} \frac{1}{2}\left[\bar{u}_{i},\left[\bar{u}^{i}, \rho\right]\right]
\end{aligned}
$$

upon selecting antisymmetric operator ordering to ensure the validity of the Lindblad properties of the evolution, and expressing the final result in terms of marginal $\bar{u}$ velocities. The (relative) negative sign in front of the entanglement term in the Liouville master equation (69) is important for exponential damping, and it was assumed in the text. Finally one identifies $\varepsilon^{2}$ with the inverse of the (Minkowski) target time $1 / t$. 
This procedure will therefore yield a master equation for Liouville decoherence in a Minkowski space-time environment of the form:

$$
\partial_{t} \rho_{\text {Matter }}=i\left[\rho_{\text {Matter }}, H\right]-\frac{\widetilde{\gamma}}{t}\left[\bar{u}_{j},\left[\bar{u}^{j}, \rho_{\text {Matter }}\right]\right]
$$

where the (positive) constant $\widetilde{\gamma}$ has been incorporated in order to take into account situations in which the density of D-particles on the brane world is less than one per Planck volume, assumed above. Lacking a detailed microscopic theory, which would in principle determine this density from first principles, we have to resort to phenomenological considerations, which are subject in principle to experimental tests. Thus, for us the parameter $0<\widetilde{\gamma}<1$ will be a free parameter to be bounded by experiment.

In the above derivation the identification $t \sim 1 / \varepsilon^{2} \rightarrow \infty$ has been used and so the form of (70) is valid for large times after the scattering of the matter string with the defect. Formally, in order to avoid unphysical singularities as $t \rightarrow 0$, we may replace the $1 / t$ term by $1 /(a+t)$, with $a$ a positive constant, i.e.

$$
\partial_{t} \rho_{\text {Matter }}=i\left[\rho_{\text {Matter }}, H\right]-\frac{\widetilde{\gamma}}{a+t}\left[\bar{u}_{j},\left[\bar{u}^{j}, \rho_{\text {Matter }}\right]\right]
$$

with $a>0$. But we stress again, that at short times after the collision our $\sigma$-model perturbation theory breaks down. It is also for this reason that the above-derived master equation is considered as rather "phenomenological".

\section{Vacuum Energy contribution to the master equation for Liouville Decoherence}

The above-described effects is not the only contribution to decoherence. If there is a vacuum energy in the space-time over which the non-critical string propagates, then the above-described foam effects will also contribute to a novel type of decoherence, associated with the vacuum energy.

To determine this effect we first notice that the renormalization-group relevant deformations (63) require Liouville dressing in order to restore the conformal invariance of the $\sigma$-model. There are two ways one can proceed in this matter. As we shall demonstrate below, the two approaches are physically equivalent, as far as the (perturbative) calculation of the back reaction onto space time is concerned.

Method I.

The first method concerns dressing of the boundary operator (63)

$$
V_{L, \text { boundary }}=\int_{\partial \Sigma} e^{\alpha_{i} \phi} y_{i}(t) \partial_{n} X^{i}, \quad \alpha_{i}=-\frac{Q}{2}+\sqrt{\frac{Q^{2}}{2}+\left(1-h_{i}\right)}
$$

where $h_{i}$ is the boundary conformal dimension, and $Q^{2}$ is the induced central charge deficit on the boundary of the world-sheet. In what follows we deal first with Euclidean targetspaces prior to Liouville dressing. 
The rate of change of $Q^{2}$ with respect to world-sheet scale $\mathcal{T} \equiv \ln |L / a|^{2} \sim \epsilon^{-2}$ is given by means of Zamolodchikov's c-theorem [26], and it is found to be of order [13] $\bar{u}_{i}^{2} \epsilon^{4}$, as being proportional to the square of the renormalization-group $\beta^{i}$ functions $\left(i=u_{i}\right)$ : $\frac{\partial Q^{2}}{\partial \mathcal{T}} \propto-\beta^{i} \mathcal{G}_{i j} \beta^{j}$, where $\mathcal{G}_{i j}=\frac{1}{\varepsilon^{2}} \delta_{i j}+\ldots$, is the Zamolodchikov metric in coupling constant $u$-space. This implies that $Q^{2}(t)=Q_{0}^{2}+\mathcal{O}\left(\epsilon^{2}\right)$, where $Q_{0}^{2}$ is constant.

We shall distinguish two cases for $Q_{0}$. The first concerns the case where $Q_{0} \neq 0$ (and by appropriate normalization may be assumed to be of order $\mathcal{O}(1)$ ). This is the case of strings living in a non-critical space time dimension. The other pertains to the case where the only source of non-criticality is the impulse deformation, i.e. $Q_{0}=0$. In the former case, one has a Liouville dimension $\alpha_{i} \sim \epsilon^{2}$, while in the latter $\alpha_{i} \sim \epsilon$.

We next rewrite the boundary operator (72) as a bulk operator, using Stokes' theorem, and then manipulate it as follows:

$$
\begin{aligned}
& V_{L, \text { boundary }}=\int_{\Sigma} \partial_{\alpha}\left(e^{\alpha_{i} \phi} y_{i}(t) \partial^{\alpha} X^{i}\right)= \\
& =\int_{\Sigma} \alpha_{i} e^{\alpha_{i} \phi} y_{i}(t) \partial_{\alpha} \phi \partial^{\alpha} X^{i}+\int_{\Sigma} e^{\alpha_{i} \phi} \dot{y}_{i}(t) \partial_{\alpha} t \partial^{\alpha} X^{i}+\int_{\Sigma} e^{\alpha_{i} \phi} y_{i}(t) \partial^{2} X^{i}= \\
& \int_{\Sigma} \alpha_{i} e^{\alpha_{i} \phi} y_{i}(t) \partial_{\alpha} \phi \partial^{\alpha} X^{i}+\int_{\Sigma} e^{\alpha_{i} \phi} \partial_{\alpha}\left(y: i(t) \partial^{\alpha} X^{i}\right)
\end{aligned}
$$

The first term in the last line describes an off-diagonal metric contribution (in our chosen coordinate system) of the form (6):

$$
g_{0 i}=\alpha_{i} y_{i}(t)
$$

\section{Method II.}

In the second method [13], one rewrites the boundary operator (63) as a bulk total world-sheet derivative operator, and then Liouville-dresses the bulk operator i.e.

$$
V_{L, \mathrm{bulk}}=\int_{\Sigma} e^{\alpha_{i} \phi} \partial_{\alpha}\left(y_{i}(t) \partial^{\alpha} X^{i}\right), \quad \alpha_{i}=-\frac{Q}{2}+\sqrt{\frac{Q^{2}}{2}+\left(2-\Delta_{i}\right)}
$$

where $\Delta_{i}$ is the conformal dimension of the bulk operator. The central charge deficit $Q$ is of the same order $Q^{2}=Q_{0}^{2}+\mathcal{O}\left(\epsilon^{2}\right)$ as in the boundary case, which implies again that $\alpha_{i} \sim \epsilon^{2}$ if $Q_{0} \neq 0$, and $\alpha_{i} \sim \epsilon$ if $Q_{0}=0$.

For the bulk operator (75) one has:

$$
\begin{aligned}
& V_{L, \text { bulk }}=\int_{\Sigma} \partial_{\alpha}\left(e^{\alpha_{i} \phi} y_{i}(t) \partial^{\alpha} X^{i}\right)-\int_{\Sigma} \alpha_{i} e^{\alpha_{i} \phi} y_{i}(t) \partial_{\alpha} \phi \partial^{\alpha} X^{i}= \\
& =\int_{\partial \Sigma} e^{\alpha_{i} \phi} y_{i}(t) \partial_{n} X^{i}-\int_{\Sigma} \alpha_{i} e^{\alpha_{i} \phi} y_{i}(t) \partial_{\alpha} \phi \partial^{\alpha} X^{i}
\end{aligned}
$$

From the second term of the last line of (76) one obtains an induced target-space metric contribution

$$
g_{0 i}=-\alpha_{i} y_{i}(t)
$$


which differs from the induced metric (74) by an overall minus sign. The latter is innocuous, and can be absorbed in a rescaling of the coordinates. Moreover, since in the respective master equations (14) the metric appears quadratically, the sign is irrelevant in this respect.

We now mention that 24] the logarithmic algebra implies a non-trivial infrared fixed point, which in the case $Q_{0} \neq 0$ is determined by $\phi_{0}=\epsilon^{-2} \sim \ln (L / a)^{2} \rightarrow \infty$, where $\phi_{0}$ is the Liouville field world-sheet zero mode. Thus, $\alpha_{i} \phi_{0}$ is finite as $\epsilon \rightarrow 0^{+}$. Therefore, as expected from the restoration of the conformal invariance by means of the Liouville dressing, one can now take safely the infra-red limit $\epsilon \rightarrow 0^{+}$in the above expressions. It is then easy to see that one is left in both cases with the target-space metric (8)), thereby proving the equivalence of both approaches at the infrared fixed point.

In the case $Q_{0}=0$, the running central charge deficit $Q^{2}=\mathcal{O}\left(\epsilon^{2}\right)$. Recalling [9] that the above formulæ imply a rescaling of the Liouville mode by $Q \sim \epsilon$, so as to have a canonical kinetic $\sigma$-model term ${ }^{1}$, and that in this case it is the $\phi_{0} / Q$ which is identified with $\ln (L / a)^{2} \sim \epsilon^{-2}$ as pertaining to the covariant world-sheet cutoff, one observes that again $\alpha_{i} \phi$ is finite as $\epsilon \rightarrow 0^{+}$, and hence similar conclusions are reached concerning the equivalence of the two methods of Liouville dressing of the impulse operator (63).

The recoil-induced metric (74) (or, equivalently (77) ), implies novel decoherence contributions to the master equation. As discussed in the text, if there are such contributions, then they show up as entanglement contributions for the case that the string propagates in a de-Sitter background. In such a case the space-time is not Ricci flat, since the corresponding Ricci tensor reads

$$
R_{\mu \nu}=\Omega g_{\mu \nu}
$$

where $\Omega$ denotes vacuum energy, which may even be allowed to depend on cosmic time. From a $\sigma$-model view point, such backgrounds are not conformal, since the corresponding graviton $\beta$-function is precisely given by the Ricci tensor to leading order in the Reggeslope $\left(\alpha^{\prime}\right)$ perturbative expansion.From the master equation (15), then, one obtains in this case the specific master equation (14) in the text.

Combining the two types of effects, D-particle foam (71) and Vacuum energy contributions (78), one may arrive at the following master equation to be used in the text:

$$
\partial_{t} \rho_{\text {Matter }}=i\left[\rho_{\text {Matter }}, H\right]-\Omega_{\text {total }}\left[\bar{u}_{j},\left[\bar{u}^{j}, \rho_{\text {Matter }}\right]\right] .
$$

where

$$
\Omega_{\text {total }}(t)=\Omega_{0}+\frac{\widetilde{\gamma}}{a+t}+\frac{\widetilde{\Gamma}}{1+b t^{2}}
$$

with the various forms having been defined in the text.

The constant $\Omega_{0}>0$ has been added to take possible account of other types of foam, or cosmological constant contributions. It is only this constant (positive) part that is responsible for exponential damping factors in physical quantities such as oscillation probabilities.

\footnotetext{
${ }^{1}$ Notice that this rescaling becomes a trivial one in the case where $Q_{0} \neq 0$.
} 


\section{Sub-leading Ohmic-type Contributions of the D-particle Foam to the master equation}

Before closing this discussion we would like to comment briefly on the form of subleading effects (as $\varepsilon \rightarrow 0$ ) associated with the operators pertaining to the D-particle coordinates $y_{i}$ in (64). Note that the relevant world-sheet operator associated with this is $\varepsilon \Theta_{\varepsilon}(t)$.

Such terms would contribute the following environmental entanglement in the master equation (15):

$$
\begin{aligned}
& \text { Sub - leading Liouville Entanglement in Eq. (15) }= \\
& : \beta^{y_{i}} \mathcal{G}_{y_{i} y_{j}}\left[y^{j}, \rho\right]+: \beta^{y_{i}} \mathcal{G}_{y_{i} u_{j}}\left[u^{j}, \rho\right]:
\end{aligned}
$$

Such terms are additional to the leading order terms exhibited in (71) above. The components of the Zamolodchikov's metric in $\left(u_{i}, y^{i}\right)$ "space" can be found by means of explicit computation using the (logarithmic) conformal algebra of the pertinent deformations [24]. To leading order in $\varepsilon \rightarrow 0$ they read:

$$
\mathcal{G}_{y_{i} y_{j}} \sim-\varepsilon^{2} \delta_{i j}, \quad \mathcal{G}_{y_{i} u_{j}} \sim \delta_{i j}
$$

where we note the relative minus sign of the first correlator. The above expressions refer to Euclidean formalism, with $\varepsilon^{2}>0$. On using (66) and passing into exactly marginal couplings $\bar{y}_{i} \equiv \frac{y_{i}}{\varepsilon}, \bar{u}_{j} \equiv \frac{u_{j}}{\varepsilon}$, with $i, j=1, \ldots d$ spatial indices, we obtain for the entanglement terms (81) (assuming antisymmetric quantum operator ordering throughout):

$$
\text { Sub - leading Liouville Entanglement in Eq. (15) }=\frac{\varepsilon^{6}}{2} \bar{y}_{i} \bar{y}^{i}-\frac{\varepsilon^{4}}{2} \bar{y}_{i} \bar{u}^{i} .
$$

Taking into account that $\varepsilon^{-2} \sim t$ is the (Minkowski) target time, and using (15), we may rewrite the entanglement (83) as follows (again formally regularizing for short times, where our approach is not valid):

$$
\begin{aligned}
& \text { Sub - leading Liouville Entanglement in Eq. (15) }= \\
& \frac{1}{2\left(a_{1}+t^{3}\right)}\left[\bar{y}_{i},\left[\bar{y}^{i}, \rho\right]\right]-\frac{1}{2 M_{P}\left(a_{2}+t^{2}\right)} r\left[\bar{y}_{i},\left[\widehat{p}^{i}, \rho\right]\right] .
\end{aligned}
$$

where $a_{1,2}$ are appropriate positive cut-off constants, serving as regulators in the $t \rightarrow 0$ limit. Upon noting that the D-particle coordinate operator $\bar{y}^{i}$ can be identified with the (stringy) probe coordinate operator $\widehat{x}_{i}$ in the entangled state, one observes that the first term in (84) acquires a conventional ohmic form $[\widehat{x},[\widehat{x}, \rho]]$ of the type considered in (17), with a time-dependent coefficient given by (84) above. This motivates the amalgamated form of the master equation considered in this work, combining ohmic and D-particle foam effects.

The randomness assumption (16) eliminates the second term of (84), which would otherwise constitute an additional entanglement term in the master equation, of a type also encountered in customary decoherent systems. However, this term may be present 
in models of foam in which there is an ordered bulk current of D-particle defects crossing the D3 brane world, which could contribute an average recoil velocity $\left\langle u_{i}\right\rangle \neq 0$ on the three-dimensional brane world. The latter type of models would result in modified dispersion relations for low-energy probes, but these need to satisfy severe phenomenological constraints 39; however, it has been argued 40 that only photons, and probably gauge bosons, could exhibit entanglement with the D-particle foam, for purely stringy reasons that we shall not discuss in this work. These latter types of probes suffer less severe phenomenological restrictions at present.

This completes our semi-rigorous (rather phenomenological) analysis of D-particle foam effects and their rôle in inducing decoherence of quantum matter propagating in it.

\section{References}

[1] S. W. Hawking, Commun. Math. Phys. 87, 395 (1982).

[2] J. R. Ellis, J. S. Hagelin, D. V. Nanopoulos and M. Srednicki, Nucl. Phys. B 241, 381 (1984).

[3] J. R. Ellis, N. E. Mavromatos and D. V. Nanopoulos, Phys. Lett. B 293, 142 (1992) arXiv:hep-ph/9207268; J. R. Ellis, J. L. Lopez, N. E. Mavromatos and D. V. Nanopoulos, Phys. Rev. D 53, 3846 (1996) arXiv:hep-ph/9505340; P. Huet and M. E. Peskin, Nucl. Phys. B 434, 3 (1995) arXiv:hep-ph/9403257; F. Benatti and R. Floreanini, Phys. Lett. B 468, 287 (1999) arXiv:hep-ph/9910508; Nucl. Phys. B 511, 550 (1998) arXiv:hep-ph/9711240. J. Bernabeu, N. E. Mavromatos and J. Papavassiliou, Phys. Rev. Lett. 92, 131601 (2004) arXiv:hep-ph/0310180; J. Bernabeu, N. E. Mavromatos, J. Papavassiliou and A. Waldron-Lauda, arXiv:hep-ph/0506025.

[4] R. Adler et al. [CPLEAR collaboration], Phys. Lett. B 364, 239 (1995) arXiv:hep-ex/9511001.

[5] G. 't Hooft, "Holography and quantum gravity," Prepared for Advanced School on Supersymmetry in the Theories of Fields, Strings and Branes, Santiago de Compostela, Spain, 26-31 Jul 1999; L. Susskind, J. Math. Phys. 36, 6377 (1995) arXiv:hep-th/9409089.

[6] J. M. Maldacena, Adv. Theor. Math. Phys. 2, 231 (1998) [Int. J. Theor. Phys. 38, 1113 (1999)] arXiv:hep-th/9711200.

[7] M. B. Green, J. H. Schwarz and E. Witten, "Superstring Theory. Vols. 1, 2" (Cambridge Univ. Press 1987).

[8] J. Polchinski, "String theory. Vol. 2: Superstring theory and beyond,"

[9] F. David, Mod. Phys. Lett. A 3, 1651 (1988); J. Distler and H. Kawai, Nucl. Phys. B 321, 509 (1989); see also: N. E. Mavromatos and J. L. Miramontes, Mod. Phys. Lett. 
A 4, 1847 (1989); E. D’Hoker and P. S. Kurzepa, Mod. Phys. Lett. A 5, 1411 (1990); for earlier works see: E. D'Hoker and R. Jackiw, Phys. Rev. D 26, 3517 (1982), and references therein.

[10] J. R. Ellis, N. E. Mavromatos and D. V. Nanopoulos, Phys. Lett. B 293, 37 (1992) arXiv:hep-th/9207103; ; arXiv:hep-th/9805120.

[11] L. J. Garay, Int. J. Mod. Phys. A 14, 4079 (1999) arXiv:gr-qc/9911002; Phys. Rev. D 58, 124015 (1998) arXiv:gr-qc/9806047|; Phys. Rev. Lett. 80, 2508 (1998) arXiv:gr-qc/9801024.

[12] J. R. Ellis, N. E. Mavromatos and M. Westmuckett, Phys. Rev. D 70, 044036 (2004) arXiv:gr-qc/0405066; Phys. Rev. D 71, 106006 (2005) arXiv:gr-qc/0501060 ;

[13] J. Ellis, N. E. Mavromatos and D. V. Nanopoulos, Phys. Rev. D 62, 084019 (2000) gr-qc/0006004, and references therein.

[14] G. Lindblad, Commun. Math. Phys. 48, 119 (1976); R. Alicki and K. Lendi, Lect. Notes Phys. 286 (Springer Verlag, Berlin (1987)).

[15] R. M. Wald, Phys. Rev. D 21, 2742 (1980).

[16] For a recent review see: P. Langacker, arXiv:hep-ph/0411116, and references therein; for LSND results in particular, see: A. Aguilar et al. [LSND Collaboration], Phys. Rev. D 64, 112007 (2001) arXiv:hep-ex/0104049; G. Drexlin, Nucl. Phys. Proc. Suppl. 118, 146 (2003).

[17] G. Barenboim and N. E. Mavromatos, JHEP 0501, 034 (2005) arXiv:hep-ph/0404014.

[18] N. E. Mavromatos, arXiv:gr-qc/0407005, Lect. Notes Phys. (Springer), in press; arXiv:hep-ph/0402005, in Venice 2003, Neutrino oscillations, 405-433.

[19] A. G. Riess et al. [Supernova Search Team Collaboration], Astron. J. 116, 1009 (1998) arXiv:astro-ph/9805201; S. Perlmutter et al. [Supernova Cosmology Project Collaboration], Astrophys. J. 517, 565 (1999) arXiv:astro-ph/9812133.

[20] D. N. Spergel et al. [WMAP Collaboration], Astrophys. J. Suppl. 148, 175 (2003) arXiv:astro-ph/0302209.

[21] A. Upadhye, M. Ishak and P. J. Steinhardt, arXiv:astro-ph/0411803.

[22] E. Gravanis and N. E. Mavromatos, Phys. Lett. B 547, 117 (2002) arXiv:hep-th/0205298; 
[23] Some review and key references include: V. Gurarie, Nucl. Phys. B 410, 535 (1993) arXiv:hep-th/9303160; M. Flohr, Int. J. Mod. Phys. A 18, 4497 (2003) arXiv:hep-th/0111228; M. R. Gaberdiel, Int. J. Mod. Phys. A 18, 4593 (2003) arXiv:hep-th/0111260|. I. I. Kogan and N. E. Mavromatos, Phys. Lett. B 375, 111 (1996) arXiv:hep-th/9512210; J. S. Caux, I. I. Kogan and A. M. Tsvelik, Nucl. Phys. B 466, 444 (1996) arXiv:hep-th/9511134; M. R. Rahimi Tabar, A. Aghamohammadi and M. Khorrami, Nucl. Phys. B 497, 555 (1997) arXiv:hep-th/9610168; K. Sfetsos, Phys. Lett. B 543, 73 (2002) arXiv:hep-th/0206091; J. Fjelstad, J. Fuchs, S. Hwang, A. M. Semikhatov and I. Y. Tipunin, Nucl. Phys. B 633, 379 (2002) arXiv:hep-th/0201091; N. E. Mavromatos and R. J. Szabo, JHEP 0301, 041 (2003) arXiv:hep-th/0207273;

[24] I. I. Kogan, N. E. Mavromatos and J. F. Wheater, Phys. Lett. B 387, 483 (1996) arXiv:hep-th/9606102; For a review focusing on D-brane recoil, including supermembranes, see: N. E. Mavromatos, arXiv:hep-th/0407026, in Shifman, M. (ed.) et al.: From fields to strings, I. Kogan memorial Volume 2, 1257-1364. (World Sci. 2005), and references therein.

[25] E. Witten, arXiv:hep-th/9206069 Phys. Rev. D44, 44 (1991);

[26] A. B. Zamolodchikov, JETP Lett. 43 (1986) 730 [Pisma Zh. Eksp. Teor. Fiz. 43 (1986) $565]$.

[27] D. Kutasov, Mod. Phys. Lett. A 7, 2943 (1992) arXiv:hep-th/9207064.

[28] B. L. Hu, J. P. Paz and Y. Zhang, Phys. Rev. D 452843 (1992)

[29] For a concise review see: P. J. Steinhardt, Phil. Trans. Roy. Soc. Lond. A 361, 2497 (2003), and references therein. See also: S. M. Carroll, Phys. Rev. Lett. 81, 3067 (1998) arXiv:astro-ph/9806099.

[30] I. Antoniadis, C. Bachas, J. R. Ellis and D. V. Nanopoulos, Nucl. Phys. B 328, 117 (1989).

[31] J. R. Ellis, N. E. Mavromatos and D. V. Nanopoulos, arXiv:hep-th/0412240, Phys. Lett. B in press.

[32] W. G. Unruh and W. H. Zurek, Phys. Rev. D 401071 (1989)

[33] C. M. Savage and D. F. Walls, Phys. Rev. A 32, 2316 (1985)

[34] E. Lisi, A. Marrone and D. Montanino, Phys. Rev. Lett. 85, 1166 (2000) arXiv:hep-ph/0002053.

[35] F. Benatti and R. Floreanini, Phys. Rev. D 64, 085015 (2001) arXiv:hep-ph/0105303. 
[36] for the most recent analysis see : D. Morgan, E. Winstanley, J. Brunner and L. F. Thompson, arXiv:astro-ph/0412618, and references therein.

[37] T. Ohlsson, Phys. Lett. B 502, 159 (2001) arXiv:hep-ph/0012272; M. Blennow, T. Ohlsson and W. Winter, arXiv:hep-ph/0502147.

[38] S. L. Adler, Phys. Rev. D 62, 117901 (2000) arXiv:hep-ph/0005220, and references therein.

[39] For a recent review see: T. Jacobson, S. Liberati and D. Mattingly, arXiv:astro-ph/0505267 and references therein.

[40] J. R. Ellis, N. E. Mavromatos, D. V. Nanopoulos and A. S. Sakharov, Int. J. Mod. Phys. A 19, 4413 (2004) arXiv:gr-qc/0312044; Nature 428, 386 (2004) arXiv:astro-ph/0309144. 